\title{
Strange Among the Strange: The B-emission Star $\gamma$ Cassiopeiæ
}

\author{
P. Harmanec \\ Astronomical Institute of the Charles University, $V$ Holešovičkách 2, \\ CZ-180 00 Praha 8, Bohemia - Czech Republic \\ and \\ Astronomical Institute, Academy of Sciences of the Czech Republic, \\ CZ-251 65 Ondřejov, Bohemia - Czech Republic
}

\begin{abstract}
Cas (HD 5394) is an important representative of Be stars, hot B stars exhibiting Balmer and other emission lines in their spectra at certain epochs. Its emission spectrum was observed as early as in 1863 and has undergone spectacular changes since then. $\gamma$ Cas is also extremely interesting because it qualifies for several phenomenologically defined groups: it is a light and colour variable, long-term radial-velocity variable, X-ray source, IRAS source, rapid line-profile variable, a singleline spectroscopic binary and a central star of a reflection nebula.

A critical compilation of the wealth of observational data on the star, with emphasis on its time variability on several time scales, as well as an estimate of the most probable values of all basic physical properties of the object is presented. It is pointed out that in spite of a large quantity of data, systematic and well-calibrated observations are still rather rare. The present understanding of the object is put into perspective of the more general - and as yet unsolved - problem of the formation of $\mathrm{Be}$ envelopes.
\end{abstract}

\section{A Continuing Puzzle of Be stars}

It is possible to say that after a century and a half of stellar spectroscopic research, astronomers basically understand the nature and physical causes of variability for virtually all types of light and spectral variables discovered so far ... but for the Be stars. This makes the Be stars, B stars with Balmer emission lines, very challenging objects among hot stars.

Open questions to be answered include: 1 . mechanism of formation of extended envelopes causing the observed emission lines; 2 . time variability on several distinct time scales; 3 . geometry of circumstellar envelopes; 4. relation to several distinct types of pulsating and rotating $B$ stars; and 5. possible role of duplicity.

Since the most pronounced variations of Be stars usually occur on a time scale of years and decades, systematic observations are very desirable. Besides, a critical analysis of already existing data for those stars for which such obser- 
vations exist, is of utmost importance. This is why objects like the archetype Be star $\gamma$ Cas are important and worthy of continuing attention.

\section{Introducing $\gamma$ Cas}

$\gamma$ Cas (27 Cas, HR 264, HD 5394, HIP 4427, MWC 9), a B0IVe star, is one of the first two ever discovered Be stars (the other one being $\beta$ Lyr) - see Secchi (1867a,b). Thanks to its brightness $\left(V=1^{\mathrm{m}} 6^{-2} 2^{\mathrm{m}} 8\right)$ and circumpolar position for many locations on the northern hemisphere $\left(\alpha_{2000.0}=0^{\mathrm{h}} 56^{\mathrm{m}} 42.5317, \delta_{2000.0}\right.$ $=+60^{\circ} 43^{\prime} 0^{\prime \prime} 265$ ), it has often been observed since then. From the point of view of a phenomenological classification, $\gamma$ Cas is an object of many faces. It has alternately been identified as a i. light variable, ii. Be star, iii. spectral variable with long-term $V / R$ and $E / C$ changes and rapid changes in the form

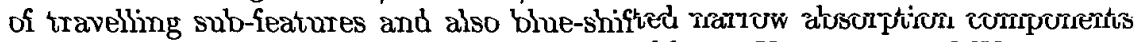
in UV resonance lines, iv. polarimetric variable, v. X-ray source MX0053+60, vi. central star of nebulæ IC 59 and IC 63 , and vii. multiple system of stars. It is a visual triple star ADS 782, with another closer component discovered via speckle interferometry plus a spectroscopic binary.

The Strasbourg CDS bibliography records more than 900 papers published on $\gamma$ Cas since 1930, and about 100 more were published earlier. From this, one could conclude that $\gamma$ Cas is a remarkably well studied object. However, a closer look reveals that it is not so easy to find studies containing either systematically collected data or pertinent analyses ...

\section{A brief observational history}

\subsection{A light variable}

Probably the first report of photometric variability was published by Birt (1832): he announced that the star varied between April 1830 and January 1831 according to his visual estimates, but gave no details. Snow (1840) reported 68 visual estimates secured between June 1839 and January 1840 , stating that $\gamma$ Cas was always brighter than either $\alpha$ Cas or $\beta$ Cas. This implies $V \leq 2{ }^{\mathrm{m}} 2$. Many visual observations were accumulated during last two decades of the $19^{\text {th }}$ century, the longest series being due to Harvard observers (see, e.g., Edwards 1944).

The first photoelectric observations of $\gamma$ Cas were obtained by Huffer (1939) who succeeded to cover a large brightening of the star, including its maximum in May 1937, related to the formation of a new envelope. Regrettably, he used another $\mathrm{Be}$ variable $\kappa$ Cas as the comparison star. Figure 1 is a time plot of $V$ magnitude of $\kappa$ Cas based on the transformed Hipparcos $H_{p}$ photometry (Perryman et al. 1997; see Harmanec 1998 for the transformation formula). The cyclic variations of $\kappa$ Cas have an amplitude of 0.1 which implies that any fine details of Huffer's light curve of $\gamma$ Cas cannot be trusted.

Since the thirties of the $20^{\text {th }}$ century, the star has been under a rather systematic patrol by both, AAVSO and BAA visual observers but the number of its photoelectric observations remains surprisingly small (see below). 

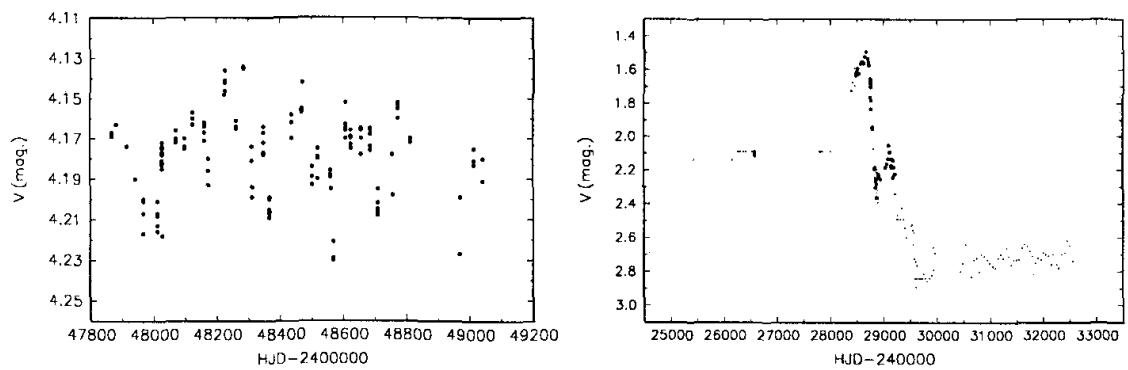

Figure 1. Left: The $V$ magnitude light curve of the Be star $\kappa$ Cas based on Hipparcos observations. Cyclic variations with a full amplitude of $0^{m} 10$ are clearly seen. Right: The $V$ magnitude light curve of $\gamma$ Cas during the 1936-1940 episode; larger symbols denote Huffer's (1939) photoelectric observations

\subsection{A spectrum variable}

Secchi $(1867 \mathrm{a}, \mathrm{b})$ observed the star with a visual spectroscope from 1863 when he saw the $\mathrm{D}_{3}=\mathrm{He}$ I 5876 line in emission. Other visual spectral observers reported large spectral variations, including a complete disappearance and reappearance of some emission lines. While it is certain that these early visual observations suffered from serious instrumental effects, I believe that their critical re-examination would still be worthy of the effort. One can argue that the reports of the presence of emission, accompanied by skillful drawings are certainly more reliable than the reports of absence of some lines which could simply be due to a worse resolution and/or seeing conditions. For instance, Konkoly's (1883) drawings - see Fig. 2 - show not only the double emission Balmer and He I lines as we know them now but obviously even stronger telluric lines in the neighbourhood of $\mathrm{H} \alpha$. Also visually observed light changes over some limit should probably be trusted.

It is true, however, that when photographic spectra of $\gamma$ Cas became available, no clear evidence of spectral changes was found (see e.g. Sidgreaves 1899; Curtiss 1916; Cleminshaw 1936; among others). The early history of investigation was skillfully reviewed by Curtiss (1916).

Talking about photographic spectra, it is worth noting that Merrill (1913) published the first intensity profiles and carried out the first attempt to detect polarized light. I digitized his $\mathrm{H} \alpha$ profile and compare it to three more recent profiles obtained with electronic detectors in Fig. 3. Note that Merrill observed what is now called a wine-bottle emission profile and was reported as a new type of the profile, only seen thanks to high $\mathrm{S} / \mathrm{N}$ ratio of electronic detectors - see Hummel \& Dachs (1992) and references therein.

Radial-velocity (RV hereafter) variations were first suspected by Hartmann (1906) but his suggestion was questioned by Campbell on the basis of Lick spectra - see Campbell \& Moore (1928). Lockyer (1933a,b) reported $V / R$ variations with about a 4-year cycle. Edwards (1944) analyzed historical spectral and photometric records and advocated the presence of a 10.67-year periodicity with two maxima, separated 4.30 years, in both, $V / R$ and brightness changes. His conclusions were challenged by Pottasch (1959) who argued that no real changes 


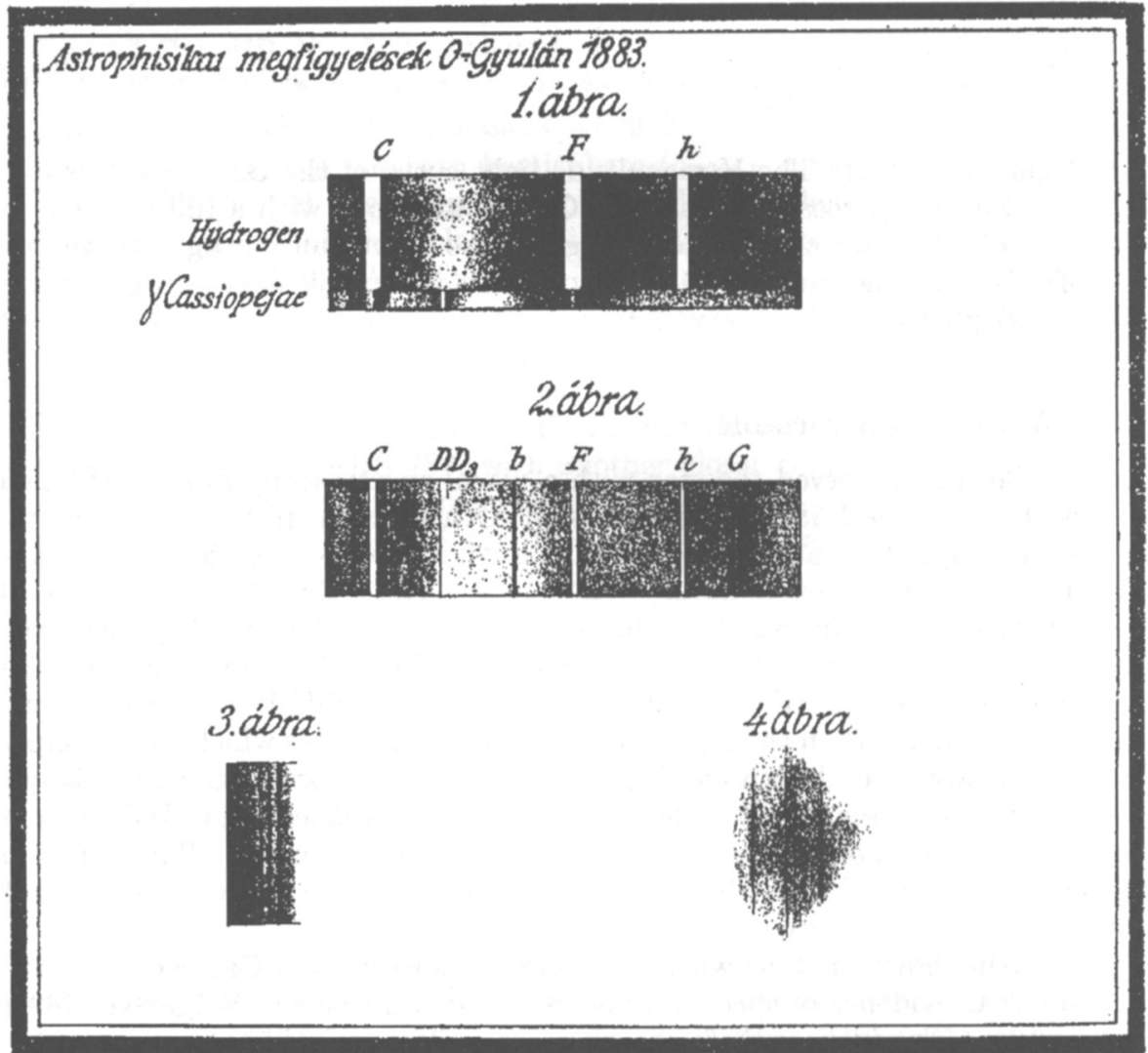

Figure 2. Konkoly's original drawings (ábra = figure in Hungarian) of the visually observed 1883 spectra of $\gamma$ Cas compared to laboratory spectrum of hydrogen, reproduced with the kind permission of the Konkoly Observatory, Hungarian Academy of Sciences. The strongest lines are identified as follows: $\mathrm{C}=\mathrm{H} \alpha, \mathrm{F}=\mathrm{H} \beta, \mathrm{h}=\mathrm{H} \gamma, \mathrm{D}=$ sodium doublet at $5889 \& 5895 \AA, D_{3}=$ He I $5876 \AA$, b = Fe II $5169 \AA$. Note that Konkoly realistically painted the double emission lines as well as stronger telluric lines near $\mathrm{H} \alpha$. 

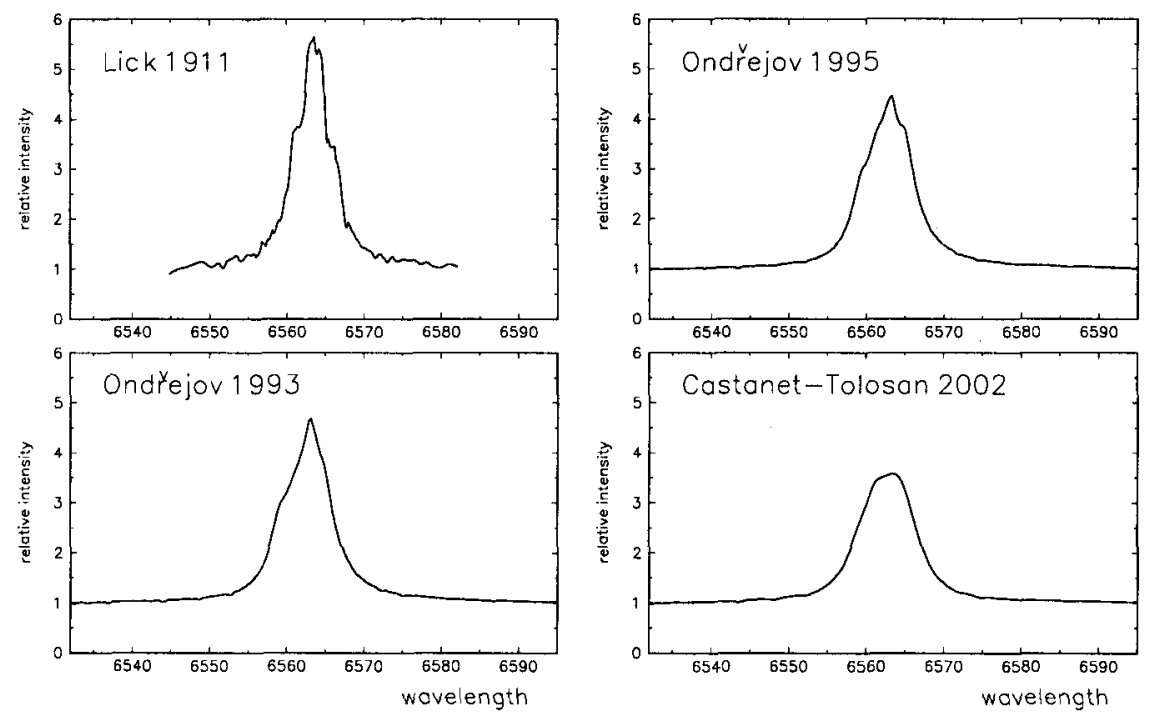

Figure 3. A comparison of $\mathrm{H} \alpha$ emission line observed by Merrill (1913) in 1911 with that from the three more recent electronic spectra

took place between 1895 and 1928. This view appears too conservative in the light of our present knowledge.

Lockyer (1935a,b, 1936) and Clemishaw (1936) were among the first to report the start of amazing spectral variations which are now known to be a large emission and two shell episodes taking place between 1936 and 1939 see, e.g., the studies by Heard (1938), Baldwin (1940), Gaze (1947), and Boyarchuk $(1958 \mathrm{a}, \mathrm{b})$. Then - for a short time - the object was almost without emission. Very regrettably, there is a lack of detailed spectral observations from that important epoch because of the war years.

Since the second half of forties, a gradual strengthening of the Balmer emission, accompanied by a mild secular brightening, has started and continued for several decades. A rather systematic monitoring of the changing Balmer emission is available thanks to photographic spectroscopy by Cowley et al. (1976), Galkina (1981; 1985; 1987; 1992; 1995), Ivanova \& Galkina (1984), Doazan et al. (1984, 1987) and Horaguchi et al. (1994).

\subsection{Rapid variations}

Probably the first report of rapid spectral variations of $\gamma$ Cas comes from Hutchings (1970) who used a high-dispersion spectral scanner in 1969 and reported rapid changes of the $\mathrm{H} \gamma$ emission profile with a 0.7 period.

Ninkov et al. (1983) discovered line-profile variations (lpv hereafter) having the form of travelling sub-features moving from blue to red across the line profiles. Their detailed account was published by Yang et al. (1988). They 
found sub-feature accelerations $a$ of $1260-2350 \mathrm{~km} \mathrm{~s}^{-1} \mathrm{~d}^{-1}$ and a mean separation of consecutive bumps of $0 \stackrel{d}{d} 091$. The presence of travelling subfeatures was independently confirmed by Horaguchi et al. (1994) who measured $a=2280$ $2580 \mathrm{~km} \mathrm{~s}^{-1} \mathrm{~d}^{-1}$ on JD 2447550 , and by Smith (1995) who found $a=2200 \pm$ $150 \mathrm{~km} \mathrm{~s}^{-1} \mathrm{~d}^{-1}$.

Measuring RVs in a series of photographic spectra, Jarad (1987) found that they vary with a period of 1.16885 . When he combined his observations with RV data by Cowley et al. (1976), he arrived at a period of 0 d 705163, close to the period reported by Hutchings, but with much higher scatter along the phase curve; this shorter period was preferred in a later study by Jarad et al. (1989). Once more, they were unable to detect any orbital RV changes.

\subsection{DAC's: Discrete absorption components in UV resonance lines}

Additional narrow, blue-shifted and variable absorption lines were discovered in several UV resonance doublets by Henrichs et al. (1983). They interpreted them as blobs of denser material in a stellar wind. Bates \& Halliwell (1986), Prinja (1988), and Harmanec $(1987,1989)$ argued that these DAC's originate from matter outflowing from local near-surface regions of a rotating star. Doazan et al. (1987) analyzed long series of optical and UV spectra and noted that DAC's are preferentially observed when $V / R>1$ and nearly absent when $V / R<1$. Their finding was confirmed by Telting \& Kaper (1994) via detailed modelling of UV spectra.

\subsection{A variable $\mathrm{X}$-ray source}

$\gamma$ Cas was identified with a new X-ray source MX0053+60 by Jernigan (1976) and this identification was confirmed by Mason et al. (1976). Shortly thereafter, Cowley et al. (1976) attempted to prove that $\gamma$ Cas is a Be $+\mathrm{X}$ binary by analyzing RVs from a large series of photographic spectra from the years 1941 -1967 . They were unable to find any RV variations with $K>20 \mathrm{~km} \mathrm{~s}^{-1}$ and a period analysis of their data detected no significant periodicity between 2.5 and $4000 \mathrm{~d}$. Besides that, Cowley et al. demonstrated that a new phase of long-term $V / R$ changes had started by $1968-69$.

Marlborough (1977) suggested that there is no binary but that the X-rays originate from bremsstrahlung in an optically thin, coronal-like region around a single star. This started a long dispute which has continued until now. Marlborough et al. (1978) actually postulated the presence of a $1 \mathrm{M}_{\odot}$ neutron-star companion in a 4-year orbit to account for the $\mathrm{X}$-ray emission and the long-term $V / R$ changes ${ }^{1}$.

White et al. (1982) argued that the X-ray spectra of $\gamma$ Cas are characteristic of a neutron star. Peters (1982) reported that a variable X-ray source had been observed since 1970 by the Uhuru satellite and that an X-ray flare was recorded by OAO3 on Jan.28, 1977. The same flare has also been detected with Ariel V (McHardy \& Pye 1981) and was followed after 3.5 hours by an increase of the $\mathrm{H} \alpha$ emission as found by Slettebak and Snow (1978). Here I ask: Is it a pure

\footnotetext{
${ }^{1}$ This model is no longer tenable not only for the physics assumed but also due to the fact that the $V / R$ variations turned out to be cyclic but certainly not periodic - see Fig. 5 .
} 
coincidence that $X$-rays were first detected at the epoch of a local brightening in $V$ and at the onset of a new epoch of $V / R$ variability which has continued up to the present time - see Fig. 5?

Dal Fiume et al. (1985) and Frontera et al. (1987) observed the star with Exosat satellite on Dec. 7, 1984 and reported a $6000 \mathrm{~s}=0.0694$ periodicity in the $\mathrm{X}$-ray flux. Note, however, that only four consecutive events during one series of observations were covered, with the two best-defined flux increases separated by 0 d 079 .

Murakami et al. (1986) recorded another large X-ray flare by the end of Oct. 1983 and suggested that the X-ray secondary is not a neutron star but a white dwarf. Further support to this idea came from analyses of ROSAT, ASCA and BeppoSAX observations - see Haberl (1995), Kubo et al. (1998) and Owens et al. (1999). In particular, Haberl (1995) - analyzing ROSAT observations - concluded that the X-ray properties of $\gamma$ Cas resemble those of magnetic cataclysmic variables. Moreover, he reported a modulation of the soft $\mathrm{X}$-ray flux with a possible periodicity of 0.094 in the $0.1-0.4 \mathrm{keV}$ flux.

I note however that the reported periods of X-ray modulation are comparable to the mean time separation of moving sub-features in the optical line profiles which - in my view - favours the origin of this modulated part of $X$-rays near the Be primary.

Myron Smith with collaborators (Cranmer, Corbet, Hatzes, Henry \& Robinson) systematically argued against the binary scenario for the X-ray emission: First, Smith (1995) analyzed Voyager 1 FUV light curves and a series of He I 6678 line profiles and estimated the rotational period of $\gamma$ Cas to be $1.04 \pm 0.17$. He noted FUV continuum light variations larger than $0^{\mathrm{m}} 2$ during less than 1 hour and pointed out that the statistics of chaotic fluctuation for FUV and X-ray curves (from Exosat and Ginga) were mutually similar. He concluded that the X-ray emission had to be associated with the Be star. Smith et al. (1998a) analyzed a simultaneous 1-d series of observations with Rossi X-ray Timing Explorer (RXTE) and High-resolution spectrograph (GHRS) on the Hubble Telescope obtained on March 14-15, 1996. They found that the UV and X-ray continuum light curves were clearly anti-correlated and estimated their possible period to be 1 d 12 . They also pointed out that the X-ray flux consists of two components, a slowly variable basal flux plus rapid flares, perhaps related to complex dynamic magnetic field structures, similar to those of cool stars. Smith et al. (1998b) added a light curve from IUE obtained over 33 hours on Jan 18-19, 1996. A comparison with the GHRS light curve gave a period of 1 . 123 . They also found variations of the EW of Si IV 1403 on the same time scale, with dips lasting only $1 / 3$ of the period. From that, they concluded that there are corotating structures some 0.2-1.9 stellar radii above the photosphere. Smith \& Robinson (1999) investigated GHRS line spectra and found moving subfeatures of cool and hot plasma in Si IV lines, similar to those seen in the optical lines. A new finding was the detection of (also narrow) absorption features with a nearly constant velocity up to $+1000-1700 \mathrm{~km} \mathrm{~s}^{-1}$ (i.e. opposite to DAC's). They argued in favour of corotating cloudlets trapped in magnetic loops. Cranmer et al. (2000) studied DAC's in Si IV from the same March 14-15, 1996 GHRS spectra found maximum absorption at $-1280 \mathrm{~km} \mathrm{~s}^{-1}$. They noted that the observations were secured near a maximum of another $V / R$ cycle and argued that mean DAC's 

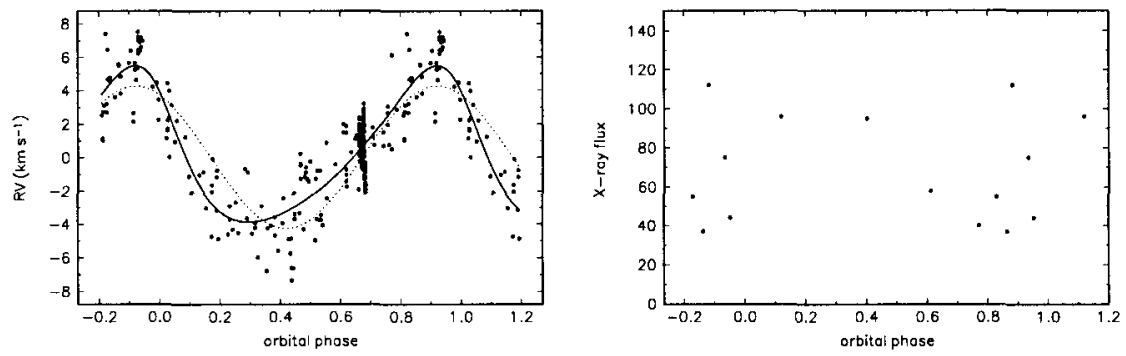

Figure 4. Left: The orbital RV curve after Harmanec et al. (2000) with the eccentric-orbit and circular-orbit solutions shown. Right: Mean X-ray flux level vs. phase after Robinson et al. (2002). In both cases, phase zero corresponds to the periastron passage for the eccentric-orbit solution, HJD 2450578.7

probably represent a broad plateau with a volume density intermediate between stellar wind and equatorial disk. According to them, subfeatures in DAC's emanate from two discrete azimuths on the star, their flickering being correlated with rotation phase. Robinson \& Smith (2000) reported a continuous 54-hour series with RXTE in Nov 24-26, 1998 plus investigation of archival IUE spectra and derived a tentative period of $1.12277 \mathrm{~d}$. They noted that the mean X-ray flux level was lower than in 1996. There is, however, little evidence for any periodicity in their $1998 \mathrm{X}$-ray light curve. In that paper, they also presented a detailed quantitative criticism of the white-dwarf scenario, showing that the accretion efficiency is too low to produce the observed X-ray flux.

\subsection{Spectroscopic binary}

Harmanec et al. (2000) derived RVs of the steep wings of the $\mathrm{H} \alpha$ emission from almost 300 red Reticon spectrograms secured between 1993 and 2000 in Ondřejov. After the removal of long-term RV changes, related to long-term cyclic $V / R$ variations, they discovered a clearly periodic RV change with a semiamplitude of only $4.7 \mathrm{~km} \mathrm{~s}^{-1}$ and a period of $203.59 \pm 0 \mathrm{~d} 29$. They also found evidence of this period in absorption-line RVs and in published data and argued that this proves the binary nature of $\gamma$ Cas. They derived an orbital solution, indicating an eccentric orbit with $e=0.26 .^{2}$ The RV curve is reproduced in Fig. 4. The orbital solution implies a probable mass of the secondary between 0.7 and $1.9 M_{\odot}$ which is plausible for a neutron star or a white dwarf secondary. However, no proof of the orbital modulation of X-rays was presented and the secondary can also be a normal late-type star.

The possibility has actually been tested by Robinson et al. (2002) who analyzed the variability of X-ray emission from nine series of RXTE observations from 1996 to 2000 along with APT $B V$ photometry secured in 4 seasons. They

\footnotetext{
${ }^{2}$ After my presentation, Dr. A.S. Miroshnichenko reported confirmation of the 204-d period for two more orbital cycles from the spectra obtained by him, Dr. Karen S. Bjorkman and their collaborators at Ritter Observatory, mentioning that their data indicate a circular orbit, however.
} 
modelled the expected flux variations on the assumption that the accretion on a putative compact companion would vary with the inverse square of the distance between the mass-losing Be star and accreting secondary and concluded that their observations have a random scatter when folded with the binary period. Instead, they found correlated cyclical optical and X-ray flux variations with 55$93 \mathrm{~d}$ cycles. From a period analysis of the nine X-ray flux data they found best sinusoidal fits for either 70.1-d or 84.4-d periods. Robinson et al. pointed out that the energy associated with the optical variations is much larger than that of the X-ray flux. They suggested that the optical and X-ray fluxes are emitted in separate volumes, with activity correlated on $50-100$-day cycles, and concluded that the most probable origin lies in a magnetic dynamo generated within the Be-star disk.

I reproduce the X-ray flux variations vs. orbital phase in Fig. 4. To my eye, the phase distribution is not completely random and it is clear that since one cannot be sure that there are no flux variations on a time scale longer than the orbital period, the result based on only 9 data points should not be accepted as the final proof to exclude any orbital modulation of the X-ray flux. While there is little doubt that a part of the X-ray flux is associated with the Be primary (as discussed above), it is still conceivable that another part of the X-ray flux originates from the secondary and varies with the orbital phase. This should further be tested though I understand that it may not be easy to get support for such satellite observations.

\subsection{Rapid optical light variations?}

There is an on-going continuing dispute on the detectability and periodicity of rapid light changes: Hipparcos $H_{p}$ photometry was analyzed by several investigators: Marchenko et al. (1998) suspected the presence of either a 1.634 or 1.045 period. Harmanec (1999) removed a linear trend present in the data and searched only in the neighbourhood of the UV light-curve period proposed by Smith et al. (1998a). He found a period of 1 d 15655. Harmanec et al. (2000) used a larger search interval and found 1.48700 , also from data prewhitened for a linear trend. Robinson et al. (2002) do not comment on the presence or absence of rapid changes in their $B V$ photometry.

\subsection{The cool end of the electromagnetic spectrum}

Coe (1986) analyzed IRAS observations of $\gamma$ Cas and suggested that the 8$22 \mu \mathrm{m}$ spectra are consistent with a blackbody at a temperature of $6750 \pm$ $100 \mathrm{~K}$. However, most of the investigators agree on the conclusion that the radiation at the cool end of the spectrum mostly comes from the circumstellar envelope - cf., e.g. Waters et al. (1991) who included also millimetre and radio observations and discussed briefly the possibility of their time variability. NearIR variability of $\gamma$ Cas has been suspected by Ferrari-Toniolo et al. (1978) and by Dougherty \& Taylor (1994). Rinehart et al. (1999) obtained the first mediumresolution 8-13.3 $\mu \mathrm{m}$ spectra and concluded that all the observed spectral lines are attributable to hydrogen recombination. Hony et al. (2000) analyzed an ISO-SWS spectrum covering $2.4-45 \mu \mathrm{m}$ and concluded that it can be fitted by a power law of $\nu^{0.99}$ over the entire range. They argued that the inner disk is 
rotating more rapidly than the star but this conclusion is probably incorrect, due to their use of an unrealistically low value of $v \sin i$ (see below).

\subsection{Polarimetry}

Linear polarization in the $\mathrm{H} \alpha$ line was detected and modelled as due to disklike envelope (cf. Poeckert \& Marlborough 1977 and references therein). Clarke (1990) analyzed Piirola's (1979) observations and found a period of 0.592 with a sinusoidal change in degree of polarization; I note that a double-wave curve would have a 1.184 period. McDavid (1999) reported his systematic $U B V R I$ polarimetric monitoring in 1995-1998. He found only marginal evidence of time variations.

\subsection{Star in a nebula}

$\gamma$ Cas is associated with moderately bright nebulæ IC 59 and IC 63 . Poeckert $\&$ Van den Bergh (1981) reported investigation of blue and red plates showing both, emission and reflection nebulæ surrounding $\gamma$ Cas; they found no change in their structure over $27 \mathrm{yr}$. They suggested that the reflection nebulosity is in the equatorial plane and that it is shielded from the ionizing Lyman continuum radiation by the dense Be envelope.

\subsection{Spectro-interferometry}

The very first angular resolution of the Be envelope was reported for $\gamma$ Cas by Thom et al. (1986), based on observations with I2T interferometer in France; they obtained an angular diameter in $\mathrm{H} \alpha$ of $0{ }^{\prime} 0052$. Quirrenbach et al. (1993) found an elliptical shape of the projected envelope detected with Mark III interferometer (which allowed a rotation of the baseline). Quirrenbach et al. (1997) obtained contemporaneous optical interferometry and spectropolarimetry of 7 stars, including $\gamma$ Cas. They found a very good agreement between the position angles of disks derived from interferometry and polarimetry, assuming polarimetric position angle to be perpendicular to the disk. According to current polarization models this rules out geometrically thick disks with large optical depths. In one case ( $\psi$ Per) they even found an agreement with radio position angle, which indicates that the spatial orientation is preserved to large distances. However, Stee et al. (1998) found a clear evidence of a lens-shaped envelope, not a disk with an opening angle, and derived angular dimensions of the object in continuum and in various spectral lines. Stee \& Bittar (2001) modelled the spectro-interferometric data and obtained better estimates of the star and disk dimensions. Berio et al. (1999) proved that the Be envelope is not axially symmetric but elongated and measured its prograde rotation correlated with the long-term $V / R$ changes.

\subsection{Summary of long-term changes}

I did my best to collect the original observations from the literature and produce homogeneous time plots showing variations of different quantitative characteristics of the line and continuum spectrum of $\gamma$ Cas. The reference time plot is shown in Fig. 5. A few remarks follow. 

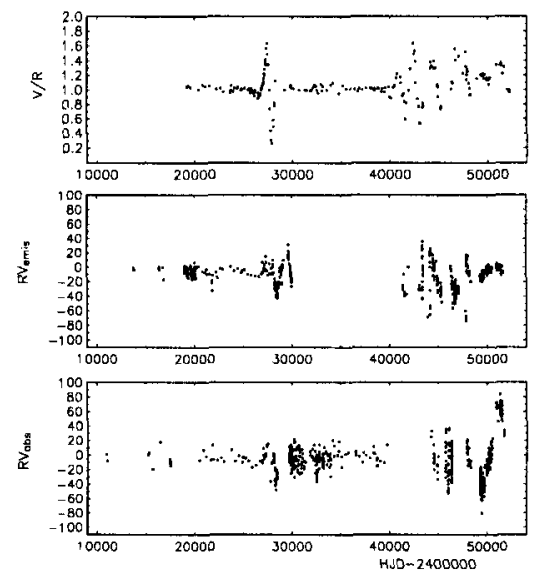
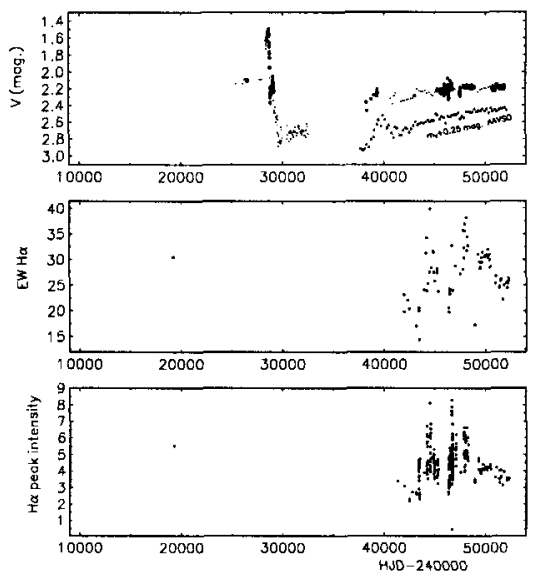

Figure 5. Long-term line and continuum variation in different observables: radial velocities of absorption and emission lines, the $V / R$ ratio of Balmer emission lines, equivalent width and peak intensity of the $\mathrm{H} \alpha$ emission and Johnson $V$ magnitude.

The optical light curve A complete one is usually showed in recent studies. However, if one tries to collect photoelectric observations which are on, or can be converted into Johnson $V$ magnitude, a remarkable lack of systematic observations is noted. Most of our knowledge rests on visual observations (shown as either 10-d or 200-d normals) and it is important to remember that the Harvard magnitude scale, used by the visual observers, differs significantly from Johnson's $V$. For $\gamma$ Cas, I found $V=m_{v i s .}-0{ }^{m} 11$ for older published data. The observations provided by AAVSO seem to develop gradually in time from the Harvard visual to Johnson's $V$ magnitude scale and cannot be directly compared to other $V$ observations. That is why I reproduce them with an arbitrary shift 0 m 25 in Fig. 5. The BAA visual observations are reduced to the Johnson's $V$, however. The most systematic recent $V$ photometry comes from Robinson et al. (2002) paper.

Spectral data RVs and spectrophotometry come from a number of publications, the longest systematic series being from the studies by Doazan with collaborators and Galkina. Also shown are the measurements from some Ondřejov Reticon spectra and the data accumulated by an amateur astronomer C. Buil. ${ }^{3}$ In cases when RV setting on the wings of the $\mathrm{H} \alpha$ emission was not published, I used the mean $\mathrm{RV}$ of the $V$ and $R$ emission peaks.

Interpretation Clearly, the non-trivial mutual relations between various observables contain important information on the nature of long-term changes. These probably arise from a combination of changes in the density and/or extent of the envelope and its gradual spatial revolution in the epochs when the

${ }^{3} \mathrm{~A}$ monotonic decrease of the $\mathrm{EW}$ of $\mathrm{H} \alpha$ between 1993 and 2001 has also been reported by a German amateur E. Pollmann but his observations are systematically shifted with respect to high-dispersion data - see Pollmann (2002). 
envelope was not axially symmetric but elongated. This caused the observed $V / R$ and $\mathrm{RV}$ changes. There has been a clear positive correlation between the brightness in $V$ and emission strength during the envelope formation, which, however, became a reverse one when the shell reached its maximum strength. This may indicate that at shell phases the envelope was almost spheroidal (which would contradict the idea of a thin disk with a small opening angle). Note also that the beginning of the second, still continuing phase of long-term $V / R$ variations, was accompanied by a temporary brightening of the object. The secular slow light increase which continued since the end of 1936-40 episode terminated a few years ago and the Balmer emission started to diminish at the same time. Does that imply that a new cycle of large spectral variations will soon begin?

\section{Basic physical properties of the object}

\subsection{Effective temperature}

It is necessary to say that estimates of the effective temperature are not trivial since both line profiles and the continuum distribution may be affected by contributions from the envelope and the temperature need not be constant over the surface of a rotating star. Some available estimates are collected in Table 1.

Table 1. Some estimates of the effective temperature and surface gravity of the B0.5 star: In column 'Method', ' $\mathrm{P}$ ' denotes values obtained from a fit to high-dispersion line profiles, ' $\mathrm{C}$ ' from modelling the continuum energy distribution (the range in nm given in brackets).

\begin{tabular}{cccl}
\hline $\begin{array}{c}T_{\text {eff. }} \\
(\mathrm{K})\end{array}$ & $\begin{array}{c}\log g \\
{[\mathrm{CGS}]}\end{array}$ & Method & Source \\
\hline $33000 \pm 3000$ & 3.73 & $\mathrm{P}$ & Hutchings (1970) \\
28000 & - & $\mathrm{P}$ & Chauville et al. (2001) \\
$32000 \pm 2500$ & $3.7 \pm 0.3$ & $\mathrm{C}(350-700)$ & Goraya (1980) \\
30000 & 3.5 & $\mathrm{C}(135-200)$ & Telting et al. (1993) \\
\hline
\end{tabular}

\subsection{Inclination}

It seems that several different methods lead to rather consistent results. Based on line-profile fitting, Hutchings (1970) derived $55^{\circ} \pm 10^{\circ}$ while Poeckert \& Marlborough (1978) arrived at $45^{\circ}$. Analyzing polarimetry, Clarke (1990) derived $43^{\circ} \pm 3^{\circ}$. From interferometry, a lower limit of $i>46^{\circ}$ was obtained by Quirrenbach et al. (1997).

\subsection{Interferometric angular diameters}

The diameter of the partly resolved envelope in the $\mathrm{H} \alpha$ emission was derived by several authors as follows: $0^{\prime \prime} 0052$ by Thom et al. (1986), $0^{\prime \prime} 0032$ by Quirrenbach et al. (1993), $0^{\prime \prime} 00347$ by Quirrenbach et al. (1997), $0^{\prime \prime} 00405$ by Stee et al. (1998), and 0.'00351 by Stee \& Bittar (2001) (via modelling).

For other spectral regions, Stee and Bittar's (2001) modelling predicts $0^{\prime \prime} .00182$ for $\mathrm{H} \beta$ emission, $0^{\prime \prime} .0009$ :: for He I $667.8 \mathrm{~nm}$ emission, 0.00969 for $\mathrm{Br} \gamma$ 
emission, $00^{\prime} 00063$ for the $480 \mathrm{~nm}$ continuum, $0{ }^{\prime} 00083$ for the $650 \mathrm{~nm}$ continuum, and $0^{\prime \prime} 00927$ for the $2160 \mathrm{~nm}$ IR continuum.

\subsection{Dereddening and parallax}

A standard dereddening based on $U B V$ photometry must fail since the envelope was present over the whole interval covered by observations and affected the observed colours of the object. I recommend using the value $E(B-V)=0^{\mathrm{m}} 07$ derived from the $220 \mathrm{~nm}$ bump and from the position in Galaxy by Beeckmans \& Hubert (1980). The best available parallax comes from Hipparcos and is 0.'00532 $\pm 0 . ' 00056$ (Perryman et al. 1997).

\subsection{Radius and mass}

There is currently no way to derive the radius and mass of the B0.5 star directly. Indirect determinations usually depend on the choice of a reliable value of $T_{\text {eff }}$. Fortunately, the dependence of the radius is weak since the change of bolometric correction with temperature nearly compensates the contribution of the temperature itself to the photometrically derived radius. Some care must be exercised to choose the visual magnitude of the star from the period when it was almost without emission and when the brightness was unaffected by a contribution from the envelope. Consulting Figs. 1 and 5 , I therefore choose $V_{\text {star }}=$ $2{ }^{\mathrm{m}} 75$ which implies a dereddened $V_{0 \mathrm{star}}=2^{\mathrm{m}} \cdot 64$. Using the above parallax and neglecting a light contribution from the secondary (no spectral lines of it were detected) one can derive a photometric (spherical) radius of $6.76-7.87 \mathrm{R}_{\odot}$. If there is a difference between the polar and equatorial radius (because of high rotation) one can assume a projected ellipse with the large axis corresponding to the equatorial radius. For $i=45^{\circ}$ one then obtains an equatorial radius of $8.0-9.4 R_{\odot}$. The mass for a given $T_{\text {eff }}$ can be estimated from Harmanec's (1988) formula calibrated via accurate determinations of masses and radii from detached eclipsing binaries.

Note that - in principle - one can carry out a consistency check using evolutionary models since for only one choice of $T_{\text {eff }}$ one obtains a radius which fits the evolutionary track for the corresponding mass in the $\log T_{\text {eff }}$ vs. $\log \mathrm{R}$ diagram. Using Schaller et al.'s (1992) evolutionary models - see Fig. 6 - one finds $M=18.3 M_{\odot}$ and $\mathrm{R}=8.0 \mathrm{R}_{\odot}$, i.e. $T_{\text {eff }}=33000 \mathrm{~K}$. This implies the angular stellar diameter of 0 ! 00040 . Note that when a new grid of evolutionary models, with rotation included, becomes available, the same procedure can return very reliable estimates of the basic physical properties of the B0.5 star.

\subsection{Linear radii and rotational velocity}

With Hipparcos parallax, one can estimate linear radii to find that the radius of the disk amounts to $71 \mathrm{R}_{\odot}$ in $\mathrm{H} \alpha$ emission and to $196 \mathrm{R}_{\odot}$ in $\mathrm{Br} \gamma$ emission. The radius of the green continuum at $480 \mathrm{~nm}$ is $13 \mathrm{R}_{\odot}$ which is larger than the stellar radius. This implies that the inner parts of the envelope act as a pseudophotosphere.

Assuming 1.487 to be the period of rotation and $i=45^{\circ}$, one finds $v \sin i$ $=385 \mathrm{~km} \mathrm{~s}^{-1}$. Note that this agrees with the $v \sin i$ observed - see Fig. 6 ; the often quoted $v \sin i=220 \mathrm{~km} \mathrm{~s}^{-1}$ after Slettebak (1982) is unrealistically low. 

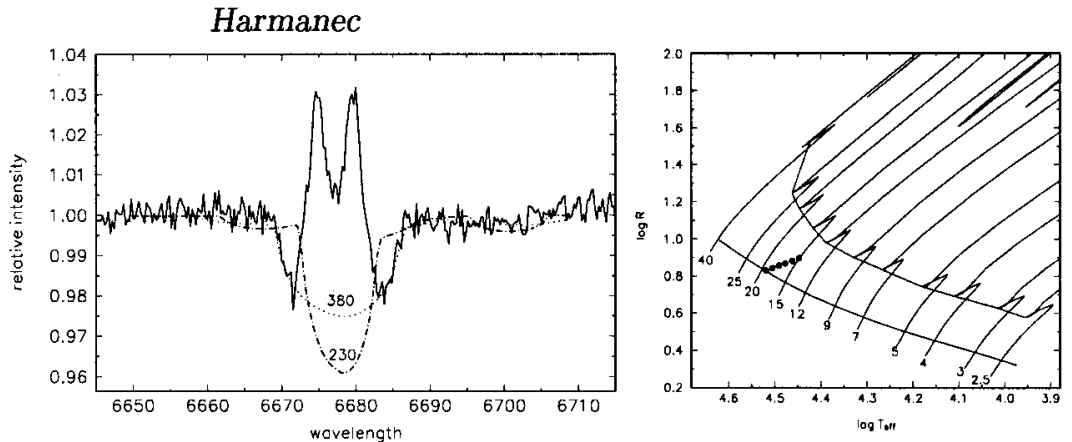

Figure 6. Left: Comparison of one 1993 Ondřejov Reticon He I 6678 line profile with synthetic spectra broadened to 230 and $380 \mathrm{~km} \mathrm{~s}^{-1}$. It is clearly seen that the photospheric wings correspond to $v \sin i=$ $380 \mathrm{~km} \mathrm{~s}^{-1}$, not to Stettebak's (1982) often quoted value of $230 \mathrm{~km} \mathrm{~s}^{-1}$. Right: A $\log R$ vs. $\log T_{\text {eff }}$ diagram with Schaller's et al. (1992) evolutionary tracks for 2.5-40 $\mathrm{M}_{\odot}$ shown. The two lines show the position of half-time between zero-age and terminal-age main sequence, and the terminal-age main sequence. Filled circles denote the position of $\gamma$ Cas for the following values of $\log T_{\text {eff }}$ and the corresponding masses (after Harmanec's (1988) formula), (4.447, 12.7), (4.462, 13.7), (4.477, 14.7), $(4.491,15.8),(4.505,17.0)$ and $(5.519,18.3)$.

\subsection{Binary properties}

Adopting also orbital inclination of $45^{\circ}$, the above primary mass and Harmanec et al. (2000) solution, one obtains: $M_{2} / M_{1}=0.07, M_{2}=1.28 \mathrm{M}_{\odot}, A=$ $392.5 \mathrm{R}_{\odot}, A_{\text {peri }}=290.4 \mathrm{R}_{\odot}$, the Roche-lobe radii being $182.5 \mathrm{R}_{\odot}$ and $51.9 \mathrm{R}_{\odot}$. Interestingly enough, the IR continuum and emission just fit into the Roche lobe around the primary.

\section{Conclusions}

I do believe that in spite of the huge number of existing studies, $\gamma$ Cas still deserves continuing systematic investigation since it is one of the crucial objects among bright Be stars and there are many not yet understood aspects of its properties and time behaviour. In particular, its X-ray properties are dissimilar to other known X-ray sources (as also pointed out by Dr. G.J. Peters during this meeting). We do not know what triggers the $V / R$ variations, have no direct information about its secondary and do not understand the nature of the rapid line-profile changes. The star should certainly become a target of future powerful spectro-interferometers.

Acknowledgments. I cordially thank to the colleagues and groups who helped me to collect and check various observational data, Drs. D. Ballereau, C.T. Bolton, C. Buil, V. Doazan, G. Henry, I. Howarth, A.-M. Hubert, S. Ilovaisky, J. Jeong, J. Mattei and the AAVSO, A. Mészáros, K. Oláh, R. Pickard and the BAA, E. Pollmann, R. Robinson, G. Scholz, M. Smith, P. Stee, and A.E. Tarasov. At the same time, I apologize to all colleagues whose work could not be mentioned explicitly because of a limited space. Drs. R.D. Robinson, 
M.A. Smith and G.W. Henry kindly gave me the permission to report the results of their study and plot their data prior to publication. I thank Chris Tout for a very careful final edit of this paper. A partial support from the organizers of the conference as well as their hospitality during the meeting are gratefully acknowledged. I profitted from the use of bibliographical service maintained at CDS Strasbourg and from the NASA ADS system. This study was supported from the research plan J13/98 113200004 and K2043105 and from program KONTAKT ME 402(2000) of MŠMT of the Czech Republic.

\section{References}

Baldwin, R. B. 1940, ApJ, 92, 82

Bates, B., \& Halliwell, D. R. 1986, MNRAS, 223, 673

Beeckmans, F., \& Hubert-Delplace, A. M. 1980, A\&A, 86, 72

Berio, P., et al. 1999, A\&A, 345, 203

Birt, W. R. 1832, MNRAS, 2, 79

Boyarchuk, A. A. 1958a, Izv. Krym, 19, 165

Boyarchuk, A. A. 1958b, Izv. Krym, 20, 118

Campbell, W. W., \& Moore, J. H. 1928, Publ. Lick Obs., 16, 1

Chauville, J., Zorec, J., Ballereau, D., Morrell, N., Cidale, L., \& Garcia, A. $2001, A \& A, 378,861$

Clarke, D. 1990, A\&A, 227, 151

Cleminshaw, C. H. 1936, ApJ, 83, 495

Coe, M. J. 1986, PASP, 98,334

Cowley, A. P., Rogers, L., \& Hutchings, J. B. 1976, PASP, 88, 911

Cranmer, S. R., Smith, M. A., \& Robinson, R. D. 2000, ApJ, 537, 433

Curtis, R. H. 1916, Publ. Astron. Obs. Univ. Michigan, 2, 1

Dal Fiume, D., Frontera, F., Manzo, G., \& Robba, R. 1985, IAU Circ., 4131

Doazan, V., Franco, M., Rusconi, L., Sedmak, G., \& Stalio, R. 1984, A\&AS, 55,1

Doazan, V., Rusconi, L., Sedmak, G., Thomas, R. N., \& Bourdonneau, B. 1987, A\&A, 182, L25

Dougherty, S. M., \& Taylor, A. R. 1994, MNRAS, 269, 1123

Edwards, D. L. 1944, MNRAS, 104, 283

Ferrari-Toniolo, M., Persi, P., \& Viotti, R. 1978, MNRAS, 185, 841

Frontera, F., Dal Fiume, D., Robba, N. R., Manzo, G., Re, S., \& Costa, E. 1987, ApJ, 320, L127

Galkina, T. S. 1981, Izv. Krym, 64, 72

Galkina, T. S. 1985 , Izv. Krym, 72, 72

Galkina, T. S. 1987, Izv. Krym, 77, 115

Galkina, T. S. 1992, Izv. Krym, 84, 32

Galkina, T. S. 1995 , Izv. Krym, 93, 102

Gaze, V. F. 1947, Izv. Krym, 1, 59 
Goraya, P. S. 1980, Ap\&SS, 73, 319

Haberl, F. 1995, A\&A, 296, 685

Harmanec, P. 1987, in IAU Coll. 92, Physics of Be Stars, ed. A. Slettebak \& T. P. Snow (Cambridge: CUP), 339

Harmanec, P. 1988, Bull. Astron. Inst. Czech., 39, 329

Harmanec, P. 1989, Bull. Astron. Inst. Czech., 40, 201

Harmanec, P. 1998, A\&A, 335, 173

Harmanec, P. 1999, A\&A, 341, 867

Harmanec, P., et al. 2000, A\&A364, L85

Hartmann, J. 1906, Astr. Nachr., 173, 102

Heard, J. F. 1938, JRASC, 32, 353

Henrichs, H. F., Hammerschlag-Hensberge, G., Howarth, I. D., \& Barr, P. 1983, ApJ, 268, 807

Hony, S., et al. 2000, A\&A, 355, 187

Horaguchi, T., et al. 1994, PASJ, 46, 9

Huffer, C. M. 1939, ApJ, 89, 139

Hummel, W., \& Dachs, J. 1992, A\&AS, 262, L17

Hutchings, J. B. 1970, MNRAS, 150, 55

Ivanova, Z. K., \& Galkina, T. S. 1984, Izv. Krym, 69, 34

Jarad, M. M. 1987, J. Space and Astron. Research (Baghdad), 4, 87

Jarad, M. M., Hilditch, R. W., \& Skillen, I. 1989, MNRAS, 238, 1085

Jernigan, J. G. 1976, IAU Circ., 2900

Konkoly, M. 1883, Magyar Tudományos Akadémia Értekezesek A Math.

Köréböl (Contr. Hungar. Acad. Sci.) No. 11, 1

Kubo, S., Murakami, T., Ishida, M., \& Corbet, R. H. D. 1998, PASJ, 50, 417

Lockyer, W. J. S. 1933a, MNRAS, 93, 362

Lockyer, W. J. S. 1933b, MNRAS, 93, 619

Lockyer, W. J. S. 1935a, MNRAS, 95, 520

Lockyer, W. J. S. 1935b, MNRAS, 96, 1

Lockyer, W. J. S. 1936, MNRAS, 96, 679

Marchenko, S. V., et al. 1998, A\&A, 331, 1022

Marlborough, J. M. 1977, ApJ, 216, 446

Marlborough, J. M., Snow, T. P., \& Slettebak, A. 1978, ApJ, 224, 157

Mason, K. O., White, N. E., \& Sanford P. W. 1976, Nature, 260, 690

McDavid, D. 1999, PASP, 111, 494

McHardy, I. M. \& Pye, J. P. 1981, Space Sci.Rev., 30, 457

Merrill, P. W. 1913, Lick Obs. Bull., 7, 162

Murakami, T., Koyama, K., Inoue, H., \& Agrawal, P. C. 1986, ApJ, 310, L31

Ninkov, Z., Yang, S., \& Walker, G. 1983, Hvar Obs. Bull., 7, 167

Owens, A., Oosterbroek, T., Parmar, A. N., Schulz, R., Stüwe, J. A., \&

Haberl, F. 1999, A\&A, 348, 170 
Parmar, A. N., Israel, G. L., Stella, L., \& White, N. E. 1993, A\&A, 275, 227

Perryman, M. A. C., Høg, E., Kovalevsky, J., Lindegren, L., \& Turon, C. 1997, ESA SP-1200: The Hipparcos and Tycho Catalogues

Peters, G. J. 1982, PASP, 94, 157

Piirola, V. 1979, A\&AS, 38, 193

Poeckert, R., \& Marlborough, M. 1977, ApJ, 218, 220

Poeckert, R., \& Marlborough, M. 1978, ApJ, 220, 940

Poeckert, R., \& van den Bergh, S. 1981, PASP, 93, 703

Pollmann, E. 2002, Be Star Newsletter, 35, 13

Pottasch, S. R. 1959, Nat. Bur. Standards, US Dept. of Commerce, Technical Note No. 21, 1

Prinja, R. K. 1988, MNRAS, 231, 21P

Quirrenbach, A., Hummel, C. A., Buscher, D. F., Armstrong, J. T., Mozurkewich, D., \& Elias II, N. M. 1993, ApJ, 416, L25

Quirrenbach, A., Bjorkman, J. E., Hummel, C. A., Buscher, D. F., Armstrong, J. T., Mozurkewich, D., Elias II, N. M., \& Babler, B. L. 1997, ApJ, 479, 477

Rinehart, S. A., Houck, J. R., \& Smith, J. D. 1999, AJ, 118, 2974

Robinson, R. D., \& Smith, M. A. 2000, ApJ, 540, 474

Robinson, R. D., Smith, M. A., \& Henry, G. W. 2002, ApJ, 575, 435

Schaller, G., Schaerer, D., Meynet, G., \& Maeder, A. 1992 A\&AS, 96, 269

Secchi, A. 1867a, Mem. Mat. Fis. Soc. Ital. Sci., $3^{\text {rd }}$ Ser., Vol. 1, Part I, 67

Secchi, A. 1867b, Astron. Nachr., 68, 63

Sidgreaves, W. 1899, MNRAS, 59, 505 \& 508

Slettebak, A. 1982, ApJS, 50, 55

Slettebak, A., \& Snow, T. P. 1978, ApJ, 224, L127

Smith, M. A. 1995, ApJ, 442, 812

Smith, M. A., \& Robinson, R. D. 1999, ApJ, 517, 866

Smith, M. A., Robinson, R. D., \& Corbet, R. H. D. 1998, ApJ, 503, 877

Smith, M. A., Robinson, R. D., \& Hatzes, A. P. 1998, ApJ, 507, 945

Snow, R. 1840, MNRAS, 5, 10

Stee, P., \& Bittar, J. 2001, A\&A, 367, 532

Stee, P., Vakili, F., Bonneau, D., \& Mourand, D. 1998, A\&A, 332, 268

Telting, J. H., \& Kaper, L. 1994, A\&A, 284, 515

Telting, J. H., Waters, L. B. F. M., Persi, P., \& Dunlop, S. R., 1993, A\&A, 270,355

Thom, C., Granes, P., \& Vakili, F. 1986, A\&A, 165, L13

Waters, L. B. F. M., van der Heuvel, W. E. C. J., Taylor, A. R., Marlborough, J. M., \& Dougherty, S. M. 1991, A\&A, 244, 120

White, N. E., Swank, J. H., \& Holt, S. S. 1982, ApJ, 263, 277

Yang, S., Ninkov, Z., \& Walker G. A. H. 1988, PASP, 100, 233 